\title{
Editorial
}

\section{Mathematical Problems in Packaging Engineering}

\author{
Jun Wang, ${ }^{1}$ Changfeng Ge, ${ }^{2}$ Dong Sun Lee, ${ }^{3}$ Michael A. Sek, ${ }^{4}$ and Vanee Chonhenchob ${ }^{5}$ \\ ${ }^{1}$ Jiangsu Province Key Laboratory of Advanced Food Manufacturing Equipment and Technology (Jiangnan University), \\ Department of Packaging Engineering of Jiangnan University, Wuxi 214122, China \\ ${ }^{2}$ Manufacturing \& Mechanical Engineering Technology/Packaging Science of Rochester Institute of Technology, \\ Rochester, NY 14623-5603, USA \\ ${ }^{3}$ Department of Food Science and Biotechnology, Kyungnam University, Changwon 631-701, Republic of Korea \\ ${ }^{4}$ School of Engineering and Science, Victoria University (VU), Melbourne, VIC 8001, Australia \\ ${ }^{5}$ Department of Packaging and Materials Technology, Faculty of Agro-Industry, Kasetsart University, Bangkok 10900, Thailand
}

Correspondence should be addressed to Jun Wang; wangj_1982@jiangnan.edu.cn

Received 19 November 2013; Accepted 19 November 2013

Copyright (C) 2013 Jun Wang et al. This is an open access article distributed under the Creative Commons Attribution License, which permits unrestricted use, distribution, and reproduction in any medium, provided the original work is properly cited.

Modern packaging plays an important role in modern industry. There are more than 60,000 scientists conducting research related to packaging engineering. In the last few decades, the packaging materials and packaging logistics have been rapidly developed that brought new challenges to mathematic modeling and simulation. This special issue aims at gathering the research and development of mathematical modeling and computation methods which have been applied into packaging engineering. Papers submitted to this special issue represent the most recent application of mathematical methods and models in the field of packaging research. Potential topics in this issue include, but are not limited to (1) novel mathematical modeling of packaging system, (2) asymptotic methods for coupled nonlinear differential equations/partial differential equations arising in packaging system, (3) fractional differential equations with applications in packaging system, (4) mathematical modeling of the mass, heat transfer phenomenon for product/packaging/human/environment interaction, (5) differential geometry with applications in packaging machinery and atomization, and (6) mathematical problems in smart packaging, active packaging, and newly developed food packaging technology.

We have solicited submissions to this special issue from packaging engineers, food engineers, mathematicians, and computer scientists. After a rigorous peer review process, 20 papers have been selected that provide the audience with overviews, solutions, and new concept for the complex packaging systems through mathematic methods. These papers have covered both the theoretical and practical aspects of complex packaging systems in the broad areas of dynamical systems, mathematics, and engineering.

Seven papers are from the research community of packaging dynamics including "Application of experimental modal analysis to determine damping properties for stacked corrugated boxes", "Drop impact analysis of cushioning system with an elastic critical component of cantilever beam type," "Application of homotopy perturbation method with an auxiliary term for nonlinear dropping equations arisen in polymer packaging system," "Study of the nonlinear dropping shock response of expanded foam packaging system," "Inner-Resonance conditions for honeycomb paperboard cushioning packaging system with critical component," "Resonance analysis for tilted support spring coupled nonlinear packaging system applying variational iteration method," and "A mathematical modeling of resonances of the nonlinear tilted support spring system under harmonic excitation." In C. Ge and S. Sutherland's work "Application of experimental modal analysis to determine damping properties for stacked corrugated boxes," the damping properties for stacked corrugated containers, which are crucial for protective packaging design, are predicted accurately by the Experimental Modal Analysis method. In D. Gao et al.s work "Drop Impact analysis of cushioning System with an elastic critical component of cantilever beam type," the 
packaged product is modeled by a cushioning system with an elastic critical component of cantilever beam type; then the dropping shock response of the system is studied. The main influencing parameters on the system's response are analyzed in details. The results obtained in this paper match well with the actual packaging system while other researchers focus more on the so-called inner-resonance conditions for different kinds of packaging system, as discussed thoroughly in "Application of homotopy perturbation method with an auxiliary term for nonlinear dropping equations arisen in polymer packaging system," "Study of the nonlinear dropping shock response of expanded foam packaging system," "InnerResonance conditions for honeycomb paperboard cushioning packaging system with critical component," "Resonance analysis for tilted support spring coupled nonlinear packaging system applying variational iteration method," and "A mathematical modeling of resonances of the nonlinear tilted support spring system under harmonic excitation."

Food packaging plays a key role in food preservation and safety, and over 45,000 researchers are engaged in some of the newer high-tech issues such as the modified atmosphere packaging, intelligent food packaging, and active packaging as well as more established novel packaging technologies. In this special issue, 7 papers from food packaging research community have been selected after a rigorous peer review process. The topics include simulation models for modified atmosphere packaging: "Applicability of simplified simulation models for perforation-mediated modified atmosphere packaging of fresh produce," "A kinetic model for predicting the relative humidity in modified atmosphere packaging and its application in Lentinula edodes packages," and "On respiratory rate of cherry tomatoes under subcritical heights"; time temperature integrator (TTI) for intelligent food packaging: "Mathematical evaluation of prediction accuracy for food quality by time temperature integrator of intelligent food packaging through virtual experiments"; and models for mass/heat transfer phenomena in food packaging system: "Study the migration process of chemical substances through the packaging/food interface during microwave treatment," "Release mathematical model of active agent from packaging material into food", and "Insulating packaging solution based on cylinder model."

Three papers from the research community of packaging materials provide us with a new insight into the recent progress in packaging materials research. In Z. Z. Sun et al.s work "The viscoelasticity model of corn straw under the different moisture contents," the viscoelasticity model of corn straw under the different moisture contents is suggested and compared with the experiment, showing perfect agreement. In J.-W. Zhou et al.s work "Application of digital image correlation to measurement of packaging material mechanical properties," a novel method, namely, the Digital Image Correlation method, is proposed to measure the mechanical properties of packaging material. While in J. Yu's research "Analysis on Nonlinear stress-growth data for shear flow of starch material with shear process," the shear flow of starch material under shear process is modeled.

S. Liu et al. reported their recent research results in packaging machinery. In Liu's work “A Decoupling control algorithm for unwinding tension system based on active disturbance rejection control," a new control methodology based on active disturbance rejection control (ADRC) for designing the tension decoupling controller of the unwinding system in a gravure printing machine is proposed. The dynamic coupling can be actively estimated and compensated in real time, which makes feedback control an ideal approach to designing the decoupling controller of the unwinding system. While in "Knowledge-based approach to assembly sequence planning for wind-driven generator," $\mathrm{M}$. Wu et al. propose the assembly connection graph (ACG) including engineering assembly semantics, which provides an appropriate way to express both geometric information and nongeometric knowledge.

In "Utilizing an adaptive grey model for short-term time series forecasting: a case study of wafer-level packaging," C.-J. Chang et al. present a new adaptive model based on grey system theory to solve the small dataset forecasting problem for wafer-level packaging process. The experimental results show that the AGM $(1,1)$ model can obtain satisfactory outcomes.

\section{Acknowledgments}

We would like to thank our colleagues in the packaging community for the support and overwhelming response to the call of the paper. Finally, we would like to acknowledge the financial support of the National Natural Science Foundation of China (Grant no. 51205167), Research Fund of Young Scholars for the Doctoral Program of Higher Education of China (Grant no. 20120093120014), and Fundamental Research Funds for the Central Universities (Grant no. JUSRP51302A) for their support for some invited submissions.

Jun Wang

Changfeng $G e$

Dong Sun Lee

Michael A. Sek

Vanee Chonhenchob 


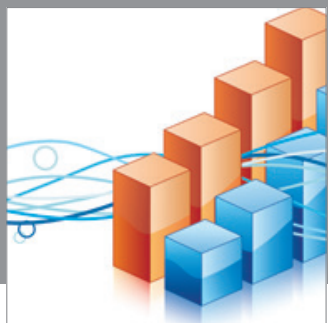

Advances in

Operations Research

mansans

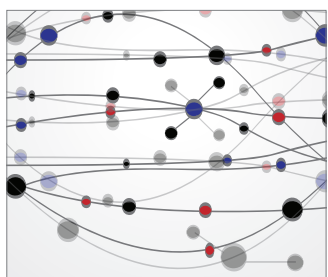

The Scientific World Journal
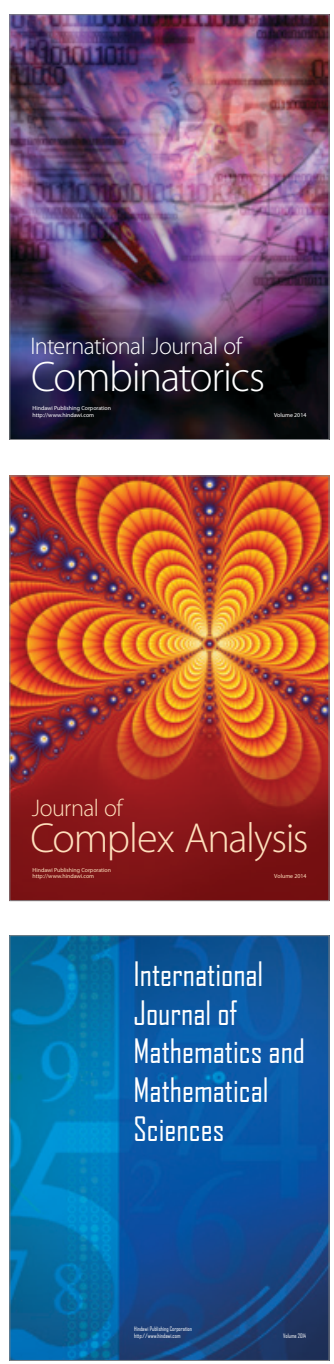
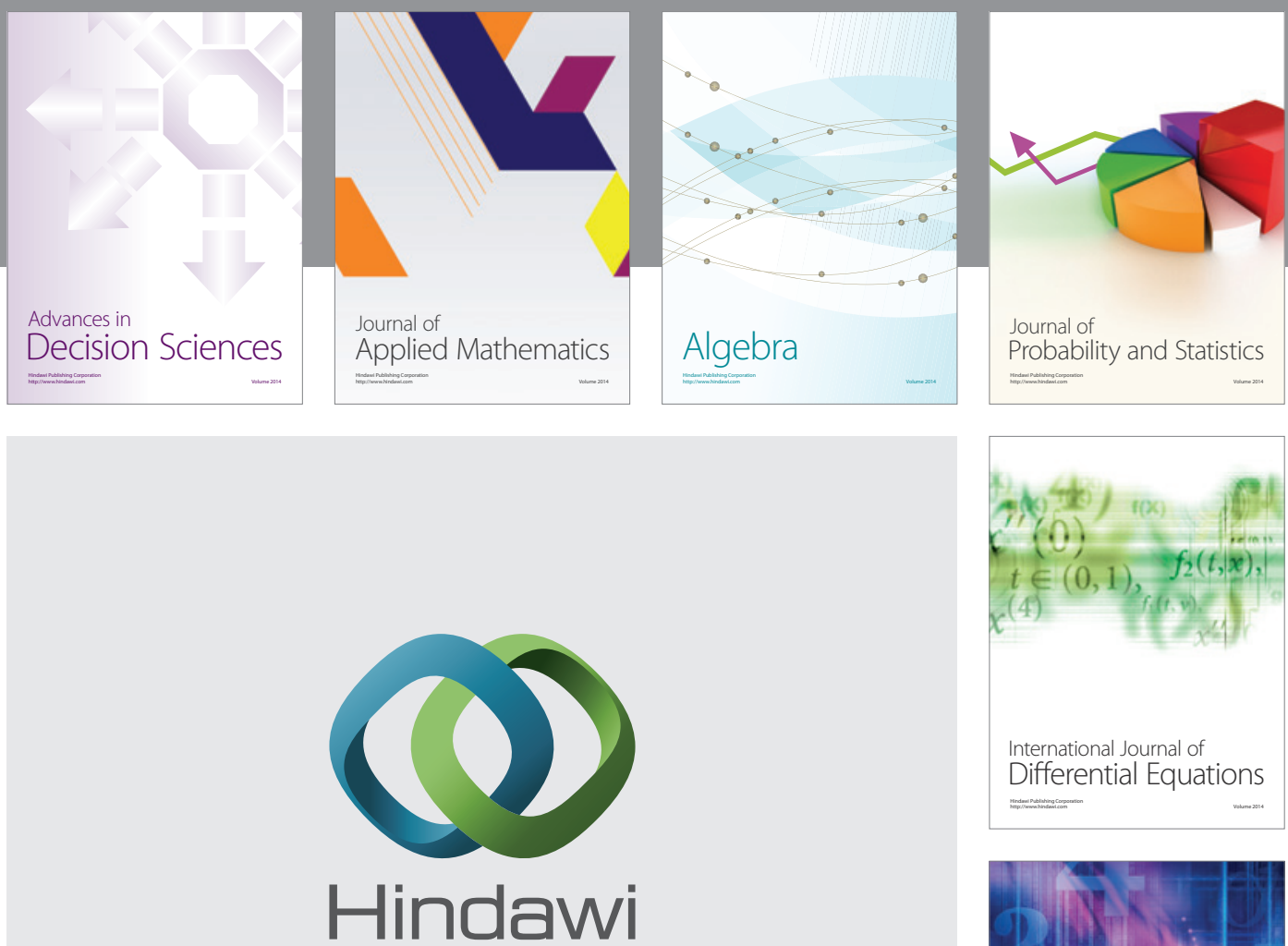

Submit your manuscripts at http://www.hindawi.com
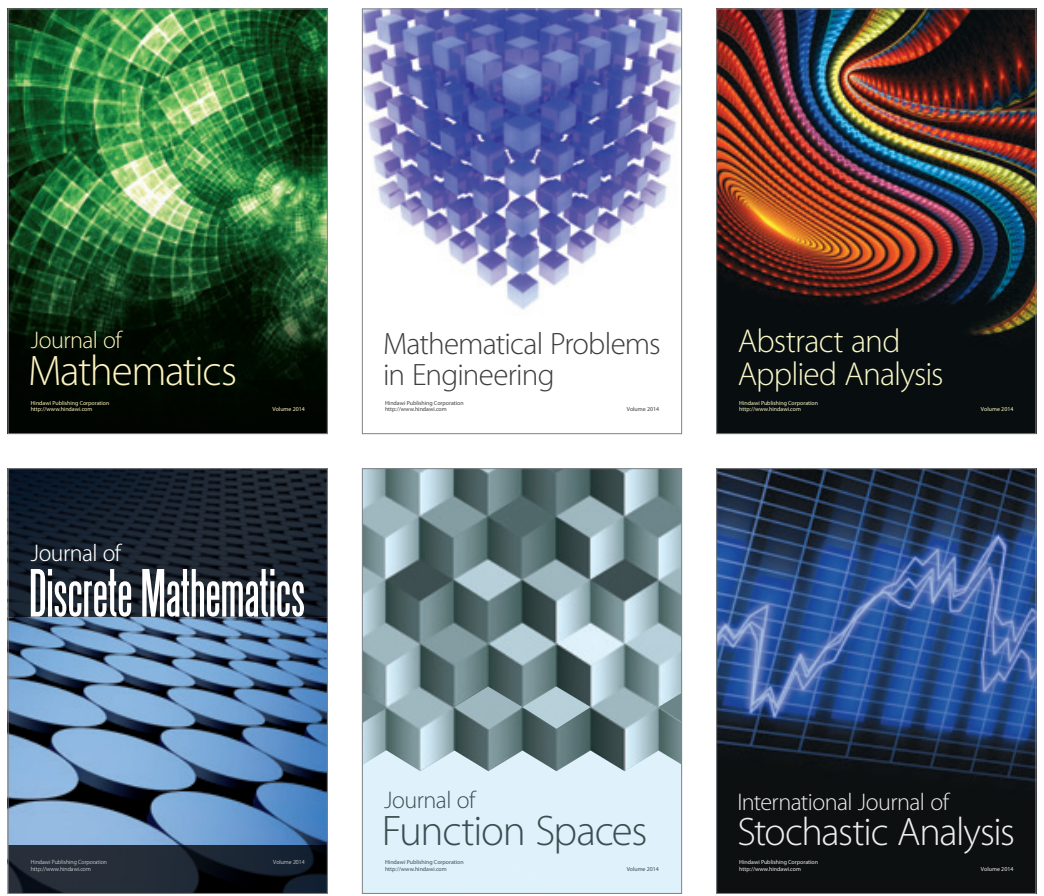

Journal of

Function Spaces

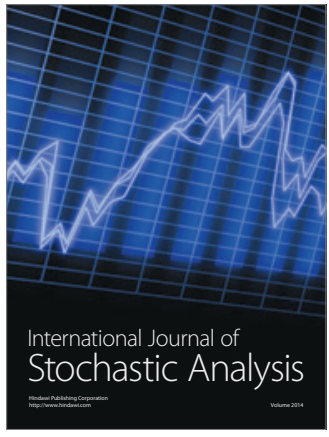

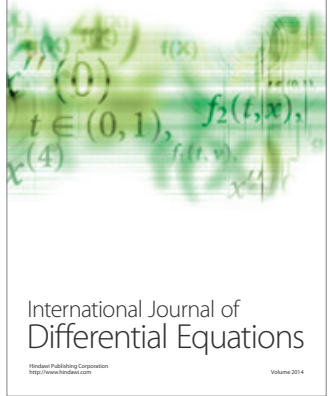
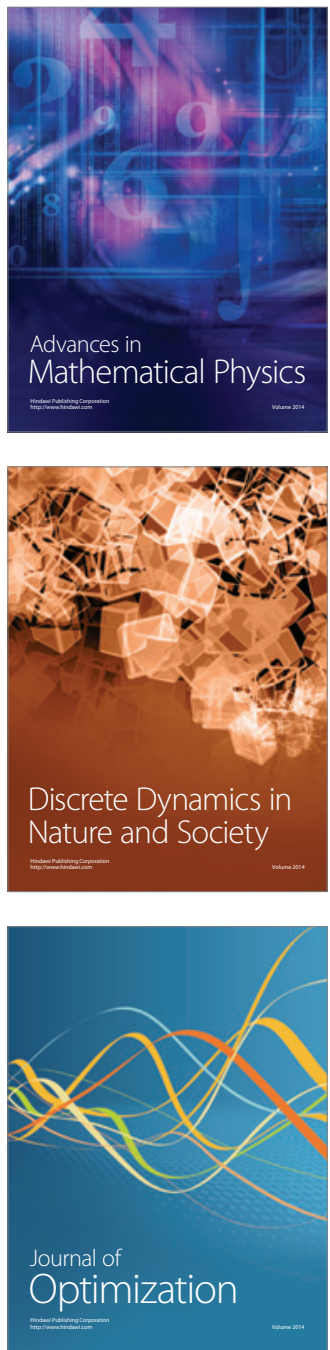